\title{
Legami capaci di futuro: dalle basi intersoggettive dello sviluppo della persona all'esperienza dello "stare" in relazione. Attaccamento, fiducia, desiderio e cura nei rapporti interpersonali
}

\author{
Gian Sandro Lerda \\ (Istituto di Psicologia Individuale “A. Adler”, Cuneo, Italy, lerda.gs@tiscali.it)
}

\section{I legami oggi}

La maggior parte dei numerosissimi studi ed approfondimenti sull'assetto e il funzionamento della società contemporanea tratteggia un quadro allarmante e scoraggiante circa lo sviluppo e il destino delle relazioni interpersonali: prevale la rappresentazione di un mondo popolato da individui narcisisticamente ripiegati su di sé ed orientati alla soddisfazione delle proprie voglie, incapaci di desiderare, costruire, mantenere legami e affetti dotati di stabilità, continuità e profondità.

A tal proposito risulta efficace la definizione di Bauman (2002) di "relazioni liquide", cioè rapporti interpersonali caratterizzati da istantaneità, brevità, transitorietà, superficialità, facile smaltibilità Relazioni che potremmo meglio definire "contatti", per utilizzare un termine molto frequente sul web, che ben rappresenta le modalità "liquide" di incontrarsi oggi. Contatti dalla vita breve, che si attivano e si spengono con facilità, mantenuti fino a che producono un beneficio, un piacere, un soddisfacimento dei propri bisogni, accostamenti fugaci che rifiutano la compenetrazione, relazioni costruite apposta per poter essere interrotte facilmente, senza fatiche o sofferenze. Perché una relazione sia capace di futuro è necessario, invece, investimento stabile, impegno, presenza, continuità, fedeltà, responsabilità Tutte esperienze che tendenzialmente oggi vengono evitate o ridotte al minimo, per superficialità o forse, andando più a fondo, per paura. Si tratta infatti, nella mentalità odierna, di esperienze potenzialmente pericolose: possono diminuire il piacere immediato, soffocare la realizzazione personale, produrre dipendenza, esporre al rischio dell'abbandono e quindi della sofferenza. Eppure le discipline psicologiche, sostenute recentemente dalle scoperte nell'ambito delle neuroscienze, concordano nel sostenere che l'esperienza relazionale e, in particolare, i legami affettivi primari siano la base della nascita e dello sviluppo psichico dell'individuo: il neonato diventa persona all'interno e in virtù della rete di relazioni nella quale nasce, vive e cresce. La relazione è dunque presupposto imprescindibile, esperienza irrinunciabile, fondante e peculiare dell'uomo. 


\section{Le basi intersoggetive dello sviluppo}

Alfred Adler, caposcuola della Individual Psicologia Comparata, affermava che "Il sentimento sociale è il barometro della normalità" (Adler 1930, 7), a sottolineare come un elemento imprescindibile e necessario per uno sviluppo sano ed una vita armonica sia proprio l'esperienza relazionale. Possiamo dire di più, sviluppando il pensiero di Adler: non solo l'esperienza relazionale è ingrediente indispensabile per un sano sviluppo ed una vita armonica, ma ne è l'elemento fondante.

Questo concetto è stato ampiamente ripreso ed elaborato da molti autori successivi in psicologia, come Winnicott, pediatra e psicoanalista, che affermava: "Un bambino da solo non esiste" (Winnicott 1951, 168), cioè non può nascere psicologicamente, come individuo, se non si trova immerso in una relazione. L'attività psichica di un bambino si sviluppa e si struttura soltanto all'interno di una relazione; invece si blocca, regredisce, si disorganizza se non è in relazione, o se questa viene a mancare o se è molto disturbata.

Tale assunto viene ripreso ed ampliato da Siegel (1999) che, introducendo il concetto di "mente relazionale", dimostra scientificamente, attraverso studi neuropsicologici, come proprio i circuiti cerebrali, le connessioni neuronali si costituiscano e si attivino sulla base delle esperienze relazionali dell'individuo. Questo processo di "costruzione della mente" si realizza in maniera massiccia durante l'infanzia e l'adolescenza, ma continua ad avvenire anche in età adulta: il cervello mantiene una certa plasticità in tutte le età della vita. Dunque è sulla base delle esperienze relazionali, e naturalmente della loro qualità e delle loro caratteristiche, che si plasma il cervello del soggetto in crescita e quindi si struttura il funzionamento neurologico e psicologico dell'individuo.

La scoperta, negli anni '90, dell'esistenza di particolari neuroni presenti nella corteccia cerebrale, chiamati neuroni a specchio (Rizzolati \& Sinigaglia 2006), ci ha dato la prova oggettiva che, non solo la mente si plasma attraverso la relazione, ma anche che il nostro cervello è predisposto alla relazione, è fatto per permetterci di entrare in relazione. I neuroni a specchio sono una classe di neuroni che si attivano quando compiamo un'azione, ma anche quando osserviamo la stessa azione compiuta da un altro soggetto: è come se anche noi facessimo quell'azione, ci identifichiamo con l'azione dell'altro. Studi ulteriori (Gallese 2007; Gallese, Keysers \& Rizzolatti 2004; Gallese 2006a) hanno evidenziato come i neuroni a specchio si attivino anche in relazione alle emozioni degli altri, espresse con la mimica facciale, i gesti, i suoni, le parole. Tali scoperte, ancora in fase di approfondimento data la complessità del fenomeno, evidenziano come il nostro cervello si attivi di fronte alle emozioni altrui e ci permetta di sentire emotivamente quello che l'altro sta vivendo: è la base dell'empatia. La capacità di entrare in empatia sarebbe dunque biologicamente determinata; sappiamo tuttavia che si svilupperà in modo più 
o meno ampio a seconda delle istanze temperamentali e delle esperienze relazionali che il soggetto farà nel corso del suo sviluppo.

\section{Le dinamiche delle relazioni primarie: sintonizzazione, riconoscimento, contenimento e costruzione del Sé}

Dunque siamo biologicamente predisposti alla relazione e ci costruiamo nella relazione. Ciò è evidente anche soltanto osservando che cosa succede tra una mamma e un bambino piccolo: fin dal momento in cui il neonato esce dalla pancia della mamma, inizia un dialogo molto intenso tra i due (che a dire il vero è già incominciato prima della nascita, nel ventre materno, un dialogo fatto di movimenti, suoni, scambi chimici intrauterini).

Già Konrad Lorenz, negli anni '40 del secolo scorso, aveva evidenziato come una serie di caratteristiche particolari del muso dei piccoli dei mammiferi, compresi i cuccioli di uomo, costituiscano il cosiddetto "prototipo infantile" (Lorenz 1949): testa grande, fronte ampia e sporgente, occhi grandi, guance paffute, forme arrotondate. Questa conformazione del volto istintivamente attrae il soggetto adulto, blocca l'aggressività e attiva schemi comportamentali di relazione e di cura nei confronti della prole.

In particolare fu poi Daniel Stern, psichiatra e psicoanalista statunitense, a studiare in modo approfondito le interazioni precoci tra mamma e bambino (Stern 1985). Egli notò come il genitore, su stimolo del prototipo infantile, istintivamente si ponga nei confronti del bambino con tipiche espressioni facciali, vocalizzi, sguardi, presentazioni del volto, comportamenti prossemici, del tutto caratteristici per modalità, frequenza, accentuazione, durata, ripetitività e diversi rispetto a quelli utilizzati nella relazione con un soggetto adulto.

Mediante tali comportamenti piuttosto accentuati la mamma capta e mantiene l'attenzione del neonato. Questo, a sua volta, possiede l'attitudine innata a riconoscere tali comportamenti sociali e risponde mettendo istintivamente in atto propri schemi comportamentali: il sorriso, i vocalizzi, i movimenti eccitati di braccia e gambe. Si viene così a creare un dialogo tra mamma e bambino, istintivamente attivato, ma che entrambi contribuiscono poi attivamente ad alimentare e a modulare. L'uno stimola l'altro in questo scambio dinamico dove si alternano momenti di eccitazione a momenti di rilassamento, momenti di avvicinamento a momenti di allontanamento, momenti di coinvolgimento a momenti di neutralità, momenti di ripetizione a momenti di novità, andando a produrre quella che Stern chiamò, con una bella espressione, la "meravigliosa danza". Ł̀ interessante notare come, fin dall'inizio, il bambino non sia passivo, bensì attivo nella relazione con la madre. 
Si avvia così, sulla base di predisposizioni innate e attraverso la ripetizione modulata di sequenze di interazione, il processo della sintonizzazione: la madre riconosce i bisogni e i sentimenti del bambino e presenta un comportamento di risposta corrispondente, atto a soddisfare tali bisogni fisiologici o affettivi; il bambino, a sua volta, riconosce la risposta materna, sperimenta soddisfazione rispetto al bisogno, collega questa esperienza a precedenti esperienze di sintonizzazione, sperimenta coerenza e continuità, sviluppa sicurezza circa il fatto di essere sintonizzato emotivamente con la madre e si sente riconosciuto. Ecco la genesi psicologica di un'esperienza evolutiva ed esistenziale fondamentale: essere riconosciuti, esperienza che permetterà poi al bambino di riconoscere se stesso. È necessario che ci sia un altro da me, in relazione con me, che mi riconosca affinché io possa riconoscere me stesso, come entità separata. Nella fusione o nell'esperienza della trascuratezza (fino all'abbandono) non c'è riconoscimento.

Il ripetersi di interazioni caratterizzate da coerenza e sintonizzazione produce nel bambino il sorgere del primo nucleo del Sé. Il genitore, attraverso le sue risposte, i suoi rimandi e i suoi rispecchiamenti, trasmette al bambino come lui lo vede e lo vive e dà un nome agli stati fisiologici ed emotivi che il bambino inizialmente sperimenta in maniera caotica ed indifferenziata (funzione del "contenimento emotivo"). Il bambino inizia così a vedere se stesso rispecchiato negli occhi della madre, a differenziare il suo mondo interno grazie all'azione di contenimento dei genitori e quindi a costruire su questa base l'immagine di sé. Possiamo dire che l'uomo conosce se stesso e costruisce la sua immagine di sé attraverso l'altro: la costruzione del Sé avviene nella relazione.

Da ciò risulta evidente come siano significative le caratteristiche dei caregiver, il loro modo di relazionarsi con il bambino e le risposte che forniscono; in seguito saranno altresì importanti le originali elaborazioni che il soggetto, crescendo, farà circa l'immagine di sé, alla luce delle successive esperienze relazionali. Possiamo dunque affermare che le esperienze relazionali precoci costituiscono la base del processo di costruzione del nucleo primario della personalità, ma la plasticità cerebrale e la continuità dell'esperienza relazionale permettono continue elaborazioni e quindi un costante evolversi della personalità anche in età successive.

\section{Corpo, linguaggio, relazione}

Non dobbiamo trascurare il fatto che un ruolo fondamentale nel processo di crescita è svolto dal corpo (Bastianini 2011). Il bambino entra in relazione con gli altri e con il mondo primariamente attraverso il corpo. L'esperienza del legame, dunque, non può prescindere dal corpo. Il bambino è corpo prima di essere pensiero e sentimenti e questi si sviluppano a partire dal corpo in 
movimento, che sperimenta. Il bambino conosce precocemente se stesso e il mondo attraverso il proprio corpo in azione e in interazione con altri corpi. Le sensazioni corporee sperimentate si depositano nella mente, si aggregano, si articolano nel tempo e ricevono dall'esterno nomi e significati, andando a costituire le prime rappresentazioni mentali di se stessi e del mondo. Esse diventano, via via, più complesse e differenziate, fino a formare un sistema rappresentazionale interno individuale che è il nostro modo originale di vedere, interpretare e vivere noi stessi e il mondo. Tutto ciò però può avvenire solo se vi è una relazione che attiva, sostiene e rinforza il processo.

È dall'esperienza relazionale corporea che sorge successivamente il linguaggio verbale. Secondo Stern, esso nasce proprio dall'esperienza della sintonizzazione: la parola è il prodotto dell'unione e del dialogo fra due corpi e due menti, che già è presente prima della parola. La parola inizialmente è un suono condiviso, che viene poi associato alla rappresentazione di un oggetto: diviene allora suono significante, che traduce verbalmente e collega le rappresentazioni mentali del bambino e del genitore. Potremmo dire che $\mathrm{i}$ bambini sviluppano il linguaggio verbale per rinforzare l'esperienza di essere con l'altro, per creare un nuovo e più efficace modo di interagire con l'altro e di condividere.

Ecco dunque come si costruisce ed evolve il legame tra genitore e figlio. Un legame tuttavia che, se si sviluppa in maniera equilibrata e sana, non contempla solo il fornire risposte gratificanti ai bisogni immediati del bambino, ma, proprio attraverso la sintonizzazione e ponendosi in una prospettiva evolutiva, impara a riconoscere i bisogni sani del bambino, quelli funzionali alla crescita e si colloca nell'ottica di fornire risposte che accompagnino, stimolino, indirizzino il bambino verso uno sviluppo armonico della sua identità e delle sue potenzialità, comprese quelle che gli permettono di entrare e rimanere in relazione con gli altri, fondamentali affinché diventi capace a sua volta di costruire e mantenere legami. Quindi non necessariamente una risposta immediatamente gratificante, ma una risposta utile alla crescita, in armonia con la realtà ed il contesto nel quale il bambino vive. Una risposta che va oltre all'individuo nel qui ed ora, ma tiene conto del contesto e di una prospettiva futura.

Possiamo dunque affermare, sulla base dei ragionamenti fin qui condotti, che le relazioni primarie, i legami affettivi di base sono presupposti imprescindibili, esperienze irrinunciabili, fondanti e strutturanti l'essere umano. La psicologia dello sviluppo, attraverso le osservazioni e le sperimentazioni, la clinica psicoterapica, attraverso lo studio e il trattamento dei disturbi e dei disagi psichici, e le neuroscienze, attraverso le recenti scoperte sulla genesi e il funzionamento del cervello, forniscono una conferma chiara e univoca rispetto a tale assunto, sicuramente già enunciato in precedenza da altre discipline. 


\section{Lo sviluppo dei legami affettivi: quattro esperienze fondamentali}

Fatte queste premesse, entriamo nel merito di quattro esperienze fondamentali che caratterizzano i legami affettivi primari e, per estensione, tutti i legami: l'attaccamento, la fiducia, il desiderio e la cura. Allo stesso tempo, proviamo a tratteggiare quali cambiamenti hanno subito queste esperienze basilari ai giorni nostri e come ciò incida sui processi di crescita, sulla costruzione della personalità e sullo sviluppo di nuovi stili relazionali.

\subsection{L'attaccamento}

Gli autori che hanno messo a punto la teoria dell'attaccamento (Bowlby, Ainsworth e altri), hanno evidenziato come i bambini, fin dalla nascita, siano naturalmente portati a sviluppare un legame nei confronti degli adulti che si occupano di loro. È evidente che una buona sintonizzazione ed esperienze reiterate di comunicazione contingente e coerente producono quello che Bowlby chiamava "attaccamento sicuro" (Bowlby 1969). L'attaccamento sicuro non solo permette al bambino di sperimentare una base solida e rassicurante a cui far riferimento, ma anche di costruire un sé solido, organizzato, stabile. Il fare esperienza di una base sicura permette al bambino di sentirsi sufficientemente tranquillo per poter affrontare la realtà con autonomia crescente. Inoltre questa esperienza relazionale primaria viene interiorizzata e diviene modello per le relazioni che il bambino andrà a costruire successivamente. Più il bambino sperimenta equilibrio, sintonia, sicurezza, stabilità, solidità, continuità nelle relazioni primarie, più sarà predisposto a sviluppare successivamente relazioni con le stesse caratteristiche.

Possiamo individuare alcune linee di cambiamento nei modelli di attaccamento, sotto l'influenza delle tendenze socio-culturali della società contemporanea?

Mediamente i genitori di oggi non mancano di attenzioni nei confronti dei bisogni che i bambini esprimono (Grandi \& Lerda 2013). Anzi molto spesso il bambino appare come un "piccolo re", le cui richieste vengono soddisfatte ancor prima di essere espresse, al riparo da ogni forma di sofferenza o mancanza. Il bambino non è più (ormai da decenni) risorsa materiale per la famiglia, da allevare come futura forza lavoro per il bene individuale e comune, ma, nella società del benessere, il figlio può rappresentare più spesso un oggetto di soddisfazione narcisistica per il genitore (o come dice Bauman [2003] un "oggetto di consumo emotivo"), oppure un prodotto di cui vantarsi, che diventa testimonianza della propria efficienza, misurata sulla base dell'agio e delle opportunità che si possono offrire, e delle competenze ed eccellenze che il bambino riuscirà a sviluppare.

Questa impronta narcisistica del legame di attaccamento primario può fare da "stampo" nel processo di costruzione del sé e da "modello" per le relazioni 
future. Più che fornire al bambino risposte coerenti ai bisogni sani di sviluppo, quindi funzionali al suo rinforzo in prospettiva futura, le risposte ruotano attorno alla soddisfazione narcisistica del genitore e del bambino. Il bambino è oggetto gratificante e soggetto da gratificare. Si sviluppa non tanto la spinta ad affrontare la vita coraggiosamente, a diventare un adulto capace e responsabile, ad inserirsi dignitosamente e produttivamente nella società, a contribuire al bene comune attraverso la capacità di riconoscere l'altro e di cooperare, quanto piuttosto l'illusione dell'onnipotenza e il bisogno di essere visto, considerato, ammirato. Su questa base anche le relazioni future tendono a costruirsi su un registro narcisistico. L'altro serve per ricevere conferme, sentirsi importanti, al centro dell'attenzione, non è un soggetto con cui condividere, completarsi, arricchirsi, progettare, costruire.

Si tratta di fatto del meccanismo che sostiene l'uso di molti social network: la vetrina, l'essere visti, la richiesta dei like. Il bisogno di essere considerati, ammirati, applauditi. Sullo stesso principio si basa il fenomeno del "sempre connessi", che testimonia la necessità di essere sempre in contatto con qualcuno, non "qualcuno in particolare", ma una "nuvola" di presenze vaga e mutevole, che conferma che ci sono, che mi guarda e mi dice qualcosa, non importa che cosa. Un'impostazione narcisistica che rivela tutta la sua fragilità e contraddittorietà: l'uomo contemporaneo non accetta legami che limiterebbero la sua libertà, che mortificherebbero la sua unicità, che lo farebbero sentire vincolato, tarpato, chiuso, ma, in realtà, ha continuamente bisogno di qualcuno che lo confermi, lo rassicuri del fatto che esiste ed è importante, è unico, è speciale, è "seguito". Mentre afferma di voler essere completamente libero ed autonomo, non "legato", non vincolato, è in realtà profondamente dipendente dagli altri e profondamente fragile: il senso di sé dipende in tutto e per tutto dalle conferme degli altri ed è dunque facilmente soggetto a oscillazioni e discontinuità. La presenza dell'altro non si connota come un legame scelto e alimentato per costruire, generare, sviluppare, ma come un contatto di cui non si può fare a meno per confermare la propria immagine, sopravvivere e placare l'angoscia del vuoto.

Osserviamo inoltre che attualmente i legami di attaccamento funzionano spesso "ad intermittenza". Quando il genitore c'è, c'è tantissimo, con un'attenzione esclusiva, quasi morbosa: non deve mancare nulla, il rapporto deve essere perfetto. Ma in altri momenti il genitore non c'è e non c'è proprio per nulla, né fisicamente né mentalmente (assorbito completamente da qualcos'altro) e viene sostituito da surrogati genitoriali. Da qui una certa discontinuità che probabilmente non contribuisce alla costruzione di quel senso di permanenza, stabilità, coerenza, continuità necessario, come abbiamo visto, per la costruzione di un sé solido e armonico. Figure di riferimento a corrente alternata, presenze che si materializzano e si smaterializzano, seguendo il meccanismo del "tutto" o "niente", possono essere all'origine di funzionamenti psicologici "per estremi": iper-stimolazione/iperattività o 
vuoto insostenibile, soddisfazione completa o frustrazione profonda, da cui può derivare un senso di valore di sé e degli altri misurato sulla base delle sensazioni momentanee di gratificazione o frustrazione, soggette quindi ad oscillazioni abissali.

Si aggiunge al quadro in esame, la crescente instabilità delle famiglie di oggi: un numero sempre in aumento di separazioni coniugali, caratterizzate spesso da un'elevata conflittualità oltre che da repentini ed imprevedibili cambiamenti in termini di tempi condivisi, spazi in cui abitare, modalità di incontro, abitudini. Assistiamo inoltre a trasformazioni significative della famiglia tradizionale, con la comparsa spesso di nuovi personaggi e la costituzione di nuovi nuclei familiari. Modificabilità, fluidità, flessibilità, precarietà, esperienze che difficilmente rispondono alle esigenze di continuità, solidità, coerenza, stabilità che abbiamo visto essere fondamentali per un soggetto in crescita.

Infine osserviamo, nella cultura contemporanea, il predominio del "fare" sullo "stare" (Grandi \& Lerda 2013): l'uomo di oggi è inserito in un vorticoso e incessante movimento che lo vede passare da un impegno all'altro, da un ambiente ad un altro, da un'attività ad un'altra, da un contatto ad un altro. Una modalità iperattiva, frammentata e discontinua, che coinvolge adulti e bambini, e non consente loro di fermarsi (forse anche per evitare l'esperienza della noia, che rimanda oggi ad una insostenibile angoscia del vuoto). Uno stile di vita che disabitua gli adulti e non permette ai bambini di abituarsi a "stare": stare in un luogo, stare in un gioco, stare ad ascoltare, stare ad osservare, stare a parlare, stare nella relazione, semplicemente godere della presenza dell'altro, dello "stare con". I genitori oggi danno molto ai loro bambini, ma tendenzialmente stanno poco con loro.

Se il maggior rischio evolutivo in passato derivava dal maltrattamento, dalla trascuratezza o dal non riconoscimento dei bisogni emotivi dei bambini nei legami di attaccamento primari, da cui si potevano sviluppare carenza di sicurezza personale, rabbia, conflitto intergenerazionale e problematiche dell'autostima, oggi il rischio più significativo è dato dall'impronta narcisistica dei legami familiari, dalla loro discontinuità e frammentarietà, da cui possono derivare centratura su di sé, fragilità identitaria, oscillazione tra opposti dell'immagine di sé e del mondo (onnipotenza/impotenza, ipervalorizzazione/iper-svalutazione), fuga dall'incontro e da se stessi attraverso l'iperattività, individualismo e dipendenza patologica.

\subsection{La fiducia}

La seconda esperienza di base che vogliamo prendere in esame è quella della fiducia. È un'esperienza così fondamentale per la crescita della persona che Erik Erikson (1950), nella sua teorizzazione sugli otto stadi dello sviluppo psicosociale dell'uomo, la pose come compito evolutivo della prima fase, tra gli 0 e i 2 anni vita. Ciò significa che il bambino, per iniziare a crescere, deve 
necessariamente sperimentare la fiducia, che costituisce la base per tutte le successive tappe evolutive.

In un'esperienza relazionale sana tra bambino e genitore, si viene a creare una concatenazione virtuosa di atti di fiducia. Innanzitutto il genitore deve coltivare buona fiducia in se stesso in quanto genitore: questo permette al bambino di sentire di potersi fidare di lui e a lui poter fare riferimento. In secondo luogo il genitore deve fidarsi del bambino e delle sue potenzialità evolutive: questo consente al bambino, a sua volta, di sviluppare fiducia in se stesso e negli altri.

Rispetto al primo punto, registriamo oggi significativi cambiamenti: i genitori si sentono tendenzialmente più insicuri, impreparati e temono spesso di non essere in grado di compiere bene il proprio compito (Di Summa 2013). Ciò che un tempo veniva espletato con naturalezza ed istintività, è oggi qualcosa per cui ci si sente carenti, bisognosi di una preparazione specifica. Come se, un tempo, il fatto stesso di diventare biologicamente genitori garantisse di essere capaci di svolgere questo ruolo. Se è vero che le minori conoscenze a livello pedagogico e psicologico e un minor grado di consapevolezza circa l'importanza del modo di prendersi cura dei bambini portava i genitori ad interpellarsi di meno circa l'educazione dei figli, è anche vero che vi era un sapere implicito che si tramandava, mansioni di cura che si imparavano naturalmente, un "occuparsi di" che faceva parte della vita familiare e riguardava anche i bambini e i ragazzi, quando un numero più elevato di figli richiedeva ai fratelli maggiori di seguire i minori. Oggi, il venir meno di queste esperienze, insieme alla maggiore complessità del mondo in cui viviamo, rendono i genitori contemporanei decisamente più confusi e disorientati di fronte ad un compito che sembra impossibile.

Ciò può produrre un atteggiamento debole del genitore di fronte al bambino, eccessivamente dubbioso e timoroso, che non trasmette solidità e sicurezza. Il bambino si sente coperto di attenzioni, di oggetti e di ansietà, ma spesso non garantito, non protetto, non guidato. Egli ha bisogno di essere ascoltato, ma anche di essere indirizzato, ha bisogno di qualcuno che gli dica che cosa si deve fare e come bisogna fare certe cose, qualcuno che gli permetta di sperimentarsi, ma che gli ponga anche dei limiti. Ha bisogno di sentire dei genitori forti, più forti di lui, in grado di sorreggerlo e di contenerlo, in grado di difenderlo anche da se stesso, da quelle spinte che lo potrebbero mettere in pericolo.

La forza e l'autorevolezza del genitore devono essere regolate e "impastate" con la fiducia che il genitore ripone nelle capacità evolutive del bambino. Se il genitore si fida delle capacità e delle peculiarità del figlio, la sua autorevolezza non si tradurrà in autoritarismo: forza, fermezza, guida, limite, ma non modalità che annulla, non riconosce, schiaccia, plasma a sua immagine $\mathrm{e}$ somiglianza. 
Avere fiducia nelle potenzialità evolutive del bambino vuol dire anche non sostituirsi a lui, lasciare che affronti le sue difficoltà, che trovi le sue soluzioni. Se una mamma prende in braccio il bambino ogni volta che piange è come se gli dicesse implicitamente: "Da solo non ce la fai, hai bisogno di me per stare bene". L'educazione viziante, molto diffusa oggi, vede il genitore impegnato ad evitare ogni difficoltà al bambino, ad eliminare possibili esperienze di frustrazione e di sofferenza, spesso sostituendosi a lui. Tale modalità non aiuta il bambino a crescere, sia perché non gli permette fattivamente di trovare proprie soluzioni ai problemi e di allenarsi a farlo, sia perché gli trasmette un messaggio di sfiducia: "Non mi fido delle tue capacità, non ci riesci da solo, lascia che faccia io". E il bambino, che si fida del genitore, pensa che sia proprio così e lascia fare, anzi a volte inizia a pretendere che siano la madre o il padre a fare le cose che dovrebbe fare lui, che intervengano subito in suo soccorso, che provvedano immediatamente a soddisfare ogni sua voglia per non dover sopportare una frustrazione. Il bambino diventa un piccolo tiranno, che nasconde, dietro la facciata arrogante e prepotente, una profonda fragilità $\mathrm{e}$ sfiducia nelle proprie capacità e risorse.

Come prima accennato infatti, l'esito della catena della fiducia è proprio la fiducia o sfiducia che il bambino sviluppa riguardo a se stesso, l'immagine di sé che costruisce. Analogamente a quanto detto riguardo all'attaccamento, sviluppare fiducia in se stessi all'interno di un legame "sicuro" consente di potersi allontanare dal porto e sperimentarsi nel mondo. Inoltre l'aver fatto esperienza di rapporti di fiducia, di rapporti che non schiacciano ma accompagnano e sostengono, permette al bambino, crescendo, di avere maggiori possibilità di fidarsi di altri che incontra sulla propria strada e di sentirsi degno di fiducia.

Fidarsi dell'altro, proprio sul modello di una relazione primaria equilibrata, non significa delegare all'altro, lasciare che l'altro faccia, o lasciarsi condizionare o schiacciare dall'altro, né pretendere da lui, ma significa decidere di condividere, permettere all'altro di entrare un po' o tanto nella propria vita, beneficiare del suo aiuto e volerlo dare senza timore di essere sfruttati o rimanere intrappolati. Una fiducia che permette di andare verso l'altro, con attenzione, con prudenza, ma anche correndo qualche inevitabile rischio, sapendo di poterlo sostenere. La fiducia in se stessi e una certa solidità del sé, permettono anche di affrontare eventuali delusioni o tradimenti, senza per questo ritirarsi in una chiusura solipsistica difensiva.

Pensare di potersi fidare solo di se stessi è un atteggiamento difensivo molto penalizzante, una scelta spesso dettata dalla paura, di fatto dalla mancanza di fiducia in se stessi, dal timore di non riuscire a sopportare la sofferenza in caso di abbandono, dall'angoscia di perdere la propria individualità e la propria libertà. Nella trincea dei muri difensivi o dall'alto del piedistallo su cui il narcisista si colloca si finisce per sperimentare situazioni di stagnazione, di 
inaridimento, di svuotamento di significato e, spesso, di malattia, come vedremo (Grandi 2002).

\subsection{II desiderio}

Ed è proprio all'interno dell'esperienza del legame che si presenta la dinamica del desiderio, il terzo fattore su cui richiamiamo la nostra attenzione. Laddove, come illustra efficacemente Recalcati (2012), il legame mantiene una connotazione fusionale-narcisistica, il bisogno è immediatamente soddisfatto $\mathrm{e}$ la madre non è persona, ma "seno", oggetto di consumo, non si sviluppa il desiderio. Perché si sviluppi il desiderio è necessaria la Legge, la Legge del Padre, il Terzo che si inserisce nella diade fusionale originaria e mette dei limiti, definisce dei confini, impone delle distanze. Allora inizia a delinearsi l'Altro, il suo esistere separato da me, con le sue caratteristiche, le sue esigenze, i suoi tempi e i suoi modi. Non è oggetto da divorare, includere, distruggere attraverso l'espressione libera delle pulsioni, ma è persona separata con cui interagire, verso cui si sviluppano sentimenti e di cui si può anche sentire la mancanza quando è lontana e desiderare il suo ritorno, il ricongiungimento.

Un legame dunque in cui si differenzia un Io e un $\mathrm{Tu}$, in cui, come dice Recalcati, "non si può avere tutto, godere di tutto, sapere tutto, essere tutto", dove si profila quindi l'eventualità dell'attesa, della frustrazione, della rinuncia, dell'impegno per raggiungere un obiettivo o per mantenere il legame stesso, del sacrificio, ma anche della creatività, dello scambio, del progetto, dell'impresa, della generatività. Senza l'esperienza del limite, non c'è veramente relazione tra un Io e un $\mathrm{Tu}$, un riconoscimento reciproco, il desiderio dell'Altro e il desiderio di essere desiderato dall'Altro, il sentire di avere un valore per l'altro e che l'altro ha valore per me, ma c'è godimento avido, individualista, centrato sull'oggetto feticcio, capriccioso, compulsivo, sregolato, privo di responsabilità, possessivo, distruttivo e autodistruttivo, di fatto anche angosciante.

Il desiderio, dunque, ha una struttura relazionale: proviene dall'Altro e si dirige verso l'Altro. Desiderio, relazione e limite sono strettamente interconnessi nello sviluppo psicologico dell'uomo. Anche rispetto a ciò, scorgiamo alcuni rischi nei modelli relazionali proposti dalla cultura contemporanea.

Charmet (2000), già alcuni anni fa, notava l'avvenuto passaggio da un modello di famiglia di tipo etico-normativo ad un modello di tipo affettivo. La famiglia etica era strutturata sul principio di autorità ed era volta a trasmettere norme e valori: principale obiettivo educativo era che i figli "si comportassero bene e trovassero un posto nella società". La famiglia affettiva di oggi, invece, è dedita primariamente a trasmettere affetto e a provvedere alla felicità dei figli: l'obiettivo è che i figli "stiano bene, siano felici, non soffrano". C'è sicuramente un guadagno dal punto di vista della disponibilità, della vicinanza emotiva, 
dell'accompagnare, del sostenere con affetto e calore, ma il rischio è che le modalità affettive possano sconfinare in stili educativi iperprotettivi o vizianti che, proprio perché non introducono limiti e norme, non stimolano il desiderio, ma forniscono appagamento immediato, godimento istantaneo, evitamento di ogni forma di frustrazione o limitazione delle possibilità. Con questo approccio educativo, faticano a svilupparsi l'intraprendenza, l'impegno, l'assunzione di responsabilità, il riconoscimento e il rispetto dell'altro come persona "altra" da me e non oggetto di gratificazione, il saper aspettare, rinunciare, tollerare la frustrazione del non avere subito e del non riuscire subito. La carenza di queste attitudini non solo penalizza, rende fragile il bambino/ragazzo di fronte ai compiti della vita, ma lo rende incapace di costruire e mantenere legami significativi nel tempo.

Questo stile educativo, inoltre, pur aspirando alla costruzione idealizzata del nido perfetto, della famiglia felice i cui membri provvedono alla reciproca gratificazione, spesso produce piccoli narcisi che finiscono per tiranneggiare anziché gratificare il genitore, portandolo talvolta a sviluppare reazioni aggressive ed espulsive.

\subsection{La cura}

Il discorso appena sviluppato ci induce ad affrontare il concetto di cura, la quarta dimensione caratterizzante i legami affettivi primari. È evidente che un essere umano per crescere ha bisogno di cure. Ma che cosa intendiamo per cura? E quali cure?

René Spitz, psicoanalista austriaco, nei suoi studi sui bambini orfani dopo la seconda guerra mondiale, aveva osservato che bambini molto piccoli si lasciavano morire o sviluppavano gravissimi sintomi depressivi anche se venivano accuditi dalle infermiere nei loro bisogni fisiologici in maniera più che adeguata (Spitz 1965). Che cosa mancava? Mancava un adulto che si prendesse "cura" di loro, nel senso più profondo del termine. Non solo cure materiali (nutrire, rispondere ai bisogni fisiologici, curare la salute) e neanche solo cure affettive generiche (fornire genericamente contatto fisico, calore, affetto), ma, come dice Luigina Mortari (2006), cure "particolari", non "anonime", cioè cure che tengano conto dell'unicità e peculiarità di quel bambino, cure che si basino sul riconoscimento dell'altro, sul desiderio dell'altro e del suo bene.

Il bambino, cioè, ha bisogno di sentire di essere nella mente del genitore. E il genitore che cura, avendo a mente il bambino e riconoscendo le sue potenzialità ad esistere, a crescere, a diventare adulto, si adopera perché vi siano le condizioni affinché queste potenzialità possano tradursi in reali capacità, il bambino possa sperimentarle, riconoscerle e svilupparle per poter "diventare quello che può essere". Non si tratta dunque di forgiare, ma di permettere al soggetto di cui ci prendiamo cura di trovare e percorrere la sua strada esistenziale. Si tratta quindi di coltivare e consentire al soggetto stesso a 
sua volta di custodire e nutrire il "desiderio di esistere", di esserci nella propria qualità unica e singolare. Si tratta anche di mantenere viva la fiducia originaria nella vita, in se stessi e negli altri; questa fiducia, anche se ben coltivata e sperimentata nelle relazioni primarie, rimane di fatto fragile di fronte alle fatiche dell'esistere e del crescere. È necessario dunque continuare a prendersene cura perché possa mantenersi e svilupparsi.

Nelle pratiche educative contemporanee assistiamo, per certi versi, ad una svalutazione del "prendersi cura" inteso in questo modo. Recentemente abbiamo più spesso sentito parlare, ad esempio in ambito scolastico, di apprendimento efficace, sviluppo delle competenze, obiettivi didattici. Quindi tecniche, strategie, modelli per aumentare l'efficacia dell'insegnamento e potenziare gli apprendimenti. Nello stesso tempo verifichiamo una crescente necessità di categorizzare, incasellare, medicalizzare: in ogni classe abbiamo gli alunni con DSA, gli iperattivi, gli autistici, gli stranieri, gli svantaggiati sociali. E i bambini dove sono finiti? È senza dubbio utile riconoscere e definire le difficoltà, gli eventuali profili psicopatologici, così come le capacità e le competenze individuali, in modo da predisporre interventi didattici finalizzati a valorizzare ed ottenere il massimo dei risultati a partire dalle potenzialità presenti in ciascuno. Ma sarebbe altresì importante che insegnanti ed educatori dedicassero energie e tempo nei loro percorsi di formazione ed aggiornamento, e poi nella loro pratica professionale, per sviluppare o potenziare la propria sensibilità e le proprie capacità di accostarsi al bambino per coglierne le sue peculiarità e modalità esistenziali, non solo le sue abilità, le sue competenze o i suoi deficit. Ciò risulta indispensabile affinché il processo educativo si sviluppi nella dimensione del "prendersi cura" e non solo del redigere documenti e piani individualizzati che stabiliscano misure dispensative e compensative e criteri di valutazione (seppur utili). Si tratta di saper cogliere e almeno tentare di entrare in sintonia con il "modo di essere al mondo" di quel bambino o di quel ragazzo, e di lavorare con lui affinché sviluppi consapevolezza di sé, delle proprie potenzialità e delle proprie difficoltà, non solo sul piano cognitivo, ma anche emotivo e relazionale, e possa coltivare fiducia nella possibilità di diventare quello che può essere.

Ma ancora una volta questo non può che passare attraverso la relazione, non attraverso un test o una tecnica educativa. Un prendersi cura che sostenga il desiderio di esserci e la fiducia del bambino in se stesso, e nello stesso tempo gli permetta di vivere l'esperienza dell'“essere con" e le potenzialità creative che si sprigionano dalla relazione.

Sentire che qualcuno si prende cura di me significa sentirmi nella mente di qualcuno, non solo nel senso che qualcuno si ricorda di me, ma anche nel senso che qualcuno mi riconosce, mi pensa, elabora qualcosa proprio per me, per come sono fatto io, per quelle che sono le mie caratteristiche e le mie possibilità di crescita. Qualcuno che parte da me, non da un'immagine ideale di me o un'immagine anonima di bambino o di persona. 
C'è da aggiungere, a proposito del discorso sulla cura e più in generale delle relazioni, che, se è vero che siamo predisposti alla relazione e che l'aver ricevuto cure apre alla possibilità di offrire cure a nostra volta, ciò non avviene automaticamente: è necessario un intervento educativo. La predisposizione alla relazione diventa capacità e pratica se viene educata. Educare alla relazione non vuol dire solo amare il bambino affinché diventi capace di amare, ma anche metterlo nelle condizioni perché si eserciti ad amare e chiedergli di farlo. Vuol dire, ad esempio, aiutarlo gradualmente a prendere coscienza che la relazione, a partire da quella con il genitore, deve andare nelle due direzioni, deve essere caratterizzata da reciprocità. E quindi portarlo a riconoscere che il genitore non è solo un dispensatore di cure, ma un soggetto che deve essere riconosciuto in quanto tale, che dunque richiede rispetto, attenzione, collaborazione. Il bambino deve essere stimolato a cooperare, ad aiutare, a mettersi al servizio a partire dall'ambiente familiare, con i genitori, con i fratelli. La connotazione narcisistica delle relazioni primarie (di cui abbiamo parlato), la tendenza alla viziatura, il contrarsi delle famiglie, sempre più isolate nel nucleo stretto e sempre più caratterizzate dalla presenza di un solo figlio, hanno notevolmente ridotto l'azione educativa, che passa attraverso la pratica quotidiana, volta a sviluppare la capacità di "prendersi cura".

Le argomentazioni finora proposte ci riportano alle premesse: da un lato abbiamo la conferma che la relazione è presupposto imprescindibile, esperienza irrinunciabile, fondante e peculiare dell'uomo, indispensabile per la sua crescita e la sua vita; dall'altro risulta evidente che i mutamenti socioculturali e le trasformazioni degli stili di vita sicuramente hanno influito ed influiscono sul modo di vivere oggi le relazioni e sulle caratteristiche dei legami. A partire dall'esame dei legami primari, su cui si struttura la personalità e che fungono da modello per le successive esperienze relazionali, ravvisiamo elementi di criticità che riguardano la continuità, la profondità, la stabilità e, ancor prima, il desiderio del legame stesso e del suo mantenimento. È messa in crisi la capacità di "stare" in relazione e di "rimanere" in relazione, di viversi in una prospettiva progettuale condivisa.

\section{6. "Stare" in relazione oggi: l'importanza di cogliere e sviluppare i segnali promettenti}

Tale analisi della società contemporanea può portare ad un certo scoraggiamento, indurre a vedere nelle attuali tendenze e nel funzionamento degli individui e dei gruppi sociali di oggi qualcosa di già deteriorato ed ancora in rapida evoluzione negativa. Allo stesso tempo tuttavia, la trattazione svolta evidenzia che, se sappiamo rivedere, riconoscere e rimettere in gioco ciò che è intrinsecamente ed essenzialmente umano fin dal momento in cui siamo concepiti, se sappiamo cogliere alcuni segnali deboli che anche le nuove 
generazioni producono e se sappiamo leggere tra le righe di alcuni eventi e comportamenti di oggi, troviamo tracce e premesse di possibili "futuri" meno catastrofici o addirittura promettenti.

Vediamo sinteticamente alcuni di questi segnali.

Facendo riferimento alla mia esperienza professionale di psicoterapeuta e ai continui confronti con i colleghi dell'Istituto di Psicologia Individuale "A. Adler", posso testimoniare che è sempre più frequente la richiesta di consulenza da parte di genitori, che si mettono autenticamente in gioco per rivedere e provare a modificare non solo il loro modo di educare, ma anche il loro modo di "stare" nelle relazioni familiari. Questo, certo, evidenzia una fragilità presente, ma anche una volontà e un impegno a lavorare su di sé.

Assistiamo anche, negli ultimi anni, ad un aumento significativo della richiesta di consulenze, incontri, conferenze, corsi sui temi della relazione educativa e, in particolare, delle regole e dei limiti, da parte di scuole, associazioni di genitori, enti di formazione. È vero che queste richieste sono dettate in prima battuta dalla difficoltà in cui si trovano i genitori e gli insegnanti alle prese con bambini ingestibili, ma contemporaneamente è senza dubbio aumentata la consapevolezza che gli stili educativi permissivi e vizianti risultano fallimentari ed ostacolano la crescita individuale e sociale, con una serie di conseguenze sul modo di vivere le relazioni e i legami di cui abbiamo parlato.

Vediamo anche, lavorando nelle scuole, che, accanto alla "medicalizzazione" e "classificazione" a cui si accennava prima, si stanno sviluppando tutta una serie di iniziative e buone prassi orientate a curare la relazione tra insegnante e allievo e a favorire la socializzazione, la cooperazione, la solidarietà, lo spirito di gruppo tra studenti, sia attraverso la costruzione di un ambiente favorevole, sia attraverso la proposta di attività esperienziali orientate (in questo la psicologia e la filosofia applicata danno importanti contributi). E la nostra esperienza ci mostra che i ragazzi colgono, si lasciano sollecitare e "risuonano" perché riconoscono la profondità di tali esperienze.

Vi sono anche persone adulte che decidono di iniziare un percorso di psicoterapia, non solo per risolvere un disturbo o liberarsi da un sintomo, anche se spesso questa è l'urgenza iniziale, ma per interrogarsi e mettersi in discussione, avvertendo che lo stile di vita sviluppato, spesso malato dal punto di vista relazionale, deve essere messo sotto la lente di ingrandimento. Gli aspetti disarmonici di sé che vanno consapevolizzati sono sempre, in qualche modo, legati alla propria storia di relazione e alle caratteristiche dei legami sperimentati. Allo stesso modo, il processo di guarigione non passa attraverso la riparazione o la sostituzione di un pezzo rotto o mancante, ma attraverso la rivisitazione e ridefinizione di sé nel rapporto con gli altri.

Nei racconti delle persone che incontriamo per lavoro o nella vita privata, inoltre, sentiamo spesso il rifiorire del desiderio di "stare con": dopo anni di tendenza alla dispersione e all'isolamento, vediamo ora spinte in controtendenza. Si moltiplicano i luoghi di ritrovo, magari meno naturali del cortile o 
della piazza di un tempo, ma "costruiti" nella forma di locali, feste, eventi culturali, manifestazioni che, tuttavia, a fianco del risvolto commerciale, denunciano un desiderio di relazione, di condivisione, di appartenenza (pur dovendo fare opportuni distinguo situazione per situazione). Allo stesso modo, il fiorire delle associazioni, delle organizzazioni di volontariato, delle iniziative umanitarie evidenziano questa tendenza (Guidi, Fonović \& Cappadozzi 2017). Se poi guardiamo al mondo giovanile, possiamo senza dubbio scorgere, tra le righe di fenomeni e comportamenti emergenti, segnali deboli ma significativi di riattivazione sul versante relazionale, che denunciano un bisogno ed un rifiorire dei legami. Da una recente ricerca del nostro Istituto sull'utilizzo della rete internet e dei social network da parte degli adolescenti e la ricaduta sul loro comportamento relazionale (Lerda \& Lo Sapio 2014) si è evidenziato che, nonostante le "distorsioni" che tali strumenti introducono, continua a confermarsi un intimo bisogno di relazione e di legame, che va riconosciuto e coltivato.

I ragazzi di oggi, pur nelle loro contraddizioni, con il loro linguaggio e subendo le influenze del nostro tempo, continuano a dimostrare lo stesso bisogno, la stessa tensione di fondo. Nella maggior parte dei casi, nonostante si muovano sul terreno instabile e liquido della nostra cultura e dei nostri stili di vita, nonostante facciano esperienze relazionali discontinue o frammentate, nonostante si facciano interpreti di modalità superficiali, anonime o narcisistiche di relazione (di fatto più semplici e di più facile consumo), opportunamente interpellati e sollecitati, ancora esprimono il bisogno di una presenza, di relazioni reali e concrete, di costruire e mantenere legami solidi, di mettersi in gioco personalmente ed emotivamente nelle relazioni e di sentire che l'altro fa lo stesso con loro, il bisogno di continuità, stabilità, sicurezza, reciprocità.

Sta a ciascuno di noi recuperare questa consapevolezza e favorire ciò che è presente ed essenziale in noi, non perché è bene fare così, perché "stare con gli altri" e mantenere legami è moralmente conveniente, ma perché noi siamo fatti così, è insito nella nostra natura, e se rinunciamo alle relazioni e ai legami, o se fuggiamo da essi, finiamo per ammalarci.

Come spesso ricorda L. G. Grandi nei suoi scritti e nei momenti di formazione, l'affermazione "io basto a me stesso" è espressione di malattia (Grandi 2016). Lo conferma anche la psicoanalista francese Ternynck, il cui celebre libro L'uomo di sabbia (2011) presenta come sottotitolo nella versione originale: "L'individualismo ci rende malati". Pensare di bastare a se stessi vuol dire aver perso il contatto con la propria interiorità e con il nucleo essenziale di sé e, allo stesso tempo, aver perso il contatto con la realtà. Bastare a se stessi è un'illusione, da cui non può che derivare insoddisfazione, malessere, solitudine, autodistruzione.

Consapevolizzare questo significa impegnarsi nella quotidianità ad "esserci" nelle relazioni con gli altri e a rivitalizzare quelle esperienze di 
sintonizzazione, attaccamento, fiducia, desiderio e cura, condizioni essenziali per una crescita sana ed una vita armonica.

\section{Conclusioni}

Il presente contributo, ripercorrendo le linee principali dei processi di sviluppo e delineando i bisogni evolutivi e le esperienze relazionali fondanti la costruzione della persona, non vuol essere soltanto una sintesi del pensiero di alcuni autori che si sono occupati di questi temi in ambito psicologico, ma si propone di evidenziare aspetti fondamentali della vita dell'uomo contemporaneo su cui orientare la nostra attenzione, le nostre riflessioni e, infine, il nostro impegno. La finalità è quella di contribuire ad alimentare una cultura e una prassi che rilancino un approccio sano alle relazioni e sostengano la possibilità di costruire ed esperire ancora legami "capaci di futuro".

Il riconoscere i condizionamenti della cultura post-moderna sulle esperienze basilari dell'attaccamento, della fiducia, del desiderio e della cura, significa acquisire strumenti di lettura critica circa i comportamenti sociali e gli atteggiamenti educativi che stanno producendo mutamenti strutturali nel funzionamento psicologico dell'uomo, con ricadute sul modo di vivere le relazioni e costruire i legami, spesso penalizzanti dal punto di vista di un armonico sviluppo individuale e di una sana realizzazione all'interno della comunità in cui viviamo.

L'ipotesi centrale di questo lavoro è che, a fronte degli stimoli esterni da cui siamo costantemente sollecitati e dei nuovi bisogni indotti ed operanti in noi consapevolmente ed inconsapevolmente, si possa lavorare a livello educativo e formativo per non perdere di vista quelli che sono i presupposti essenziali ed irrinunciabili di un percorso armonico di crescita.

Si tratta di promuovere e sostenere riflessioni ed azioni concrete che permettano di riprendere contatto e dare spazio a quei sani bisogni di relazione evidenziati nella trattazione precedente, la cui realizzazione è indispensabile non solo per il soggetto in crescita, ma anche per l'individuo adulto che intenda accedere ad un percorso di vita rispondente alle sue istanze più profonde, connotate da bisogni di condivisione, intimità e generatività.

La consapevolezza circa le conseguenze negative delle dinamiche narcisistiche, dell'instabilità dei legami familiari, della presenza intermittente dei genitori, della prevalenza del "fare" sullo "stare" nei percorsi di sviluppo (per citare alcuni degli aspetti trattati nei paragrafi precedenti) sollecita a porre attenzioni particolari nei confronti dei soggetti in crescita, al fine di rinforzare quelle esperienze che garantiscano la costruzione di legami di attaccamento caratterizzati da continuità, solidità, stabilità, reciprocità, fiducia. Tali esperienze costituiscono condizioni essenziali affinché il futuro adulto sia 
dotato della struttura psichica e degli strumenti necessari per costruire, sostenere e mantenere a sua volta relazioni e legami affettivi, indispensabili per il suo benessere. Sviluppare questa consapevolezza e agire per creare condizioni che facilitino la costruzione e la sperimentazione di relazioni educative sane, può costituire anche per l'adulto di oggi uno stimolo per la propria vita, una motivazione ed un impegno a ritrovare dentro di sé i bisogni e le spinte vitali fondamentali, che necessitano di essere espresse, alimentate, condivise.

La precarietà, l'insicurezza, la carenza di autorevolezza, la viziatura, l'indebolimento degli atteggiamenti di cura "particolare" (per citare altri aspetti affrontati in questo scritto) che permeano gli atteggiamenti e le pratiche educative odierne, devono essere portati a consapevolezza e problematizzati, al fine di stimolare processi di cambiamento attraverso opportuni interventi formativi. Riscoprire, ad esempio, il valore e l'utilità dei limiti e delle regole, pur tenendo conto della complessità e varietà degli attuali contesti, oppure coltivare un approccio alla persona che non isoli le parti e potenzi le prestazioni, ma si prenda cura della globalità e della specificità del soggetto inserito nella comunità, sono esempi di azioni educative e formative che agiscono contro tendenza (di cui vediamo già importanti segnali oggi) e che risultano più che mai potenti nei processi di accompagnamento alla crescita perché non rimangono sul piano astratto della teoria o dello slogan, ma si sviluppano nel concreto dell'esperienza relazionale (che diviene così, nel contempo, oggetto e strumento degli interventi stessi), rispondendo ai bisogni più profondi dell'uomo e riattivando quel potenziale creativo che permette di guardare con speranza al domani.

In estrema sintesi di tratta di riconoscere, per poter rilanciare e coltivare, l'essenzialità e la potenza degli affetti e dei legami, ingredienti fondamentali ed irrinunciabili nella progettazione di una qualsiasi idea di futuro umanamente sostenibile. Estrapolando le parole del personaggio Titta di Girolamo nel film di Sorrentino Le conseguenze dell'amore (2004), potremmo dire: "Progetti per il futuro? Non sottovalutare le conseguenze dell'amore".

\section{Opere citate}

Adler, A. 1930. The Education of Children. Trad. it. Psicologia dell'educazione. Roma: Newton Compton (1975).

Bastianini, A. M. 2011. Psicoterapia infantile: una prospettiva di sviluppo per la pratica clinica adleriana, in: Il Sagittario, 28, 33-44.

Bauman, Z. 2002. Liquid Modernity. Trad. it: Modernità Liquida. Bari: Laterza (2011).

Bauman, Z. 2003. Liquid Love. On the Frailty of Human Bonds. Trad. it. Amore liquido. Sulla fragilità dei legami affettivi. Bari: Laterza (2006). 
Bowlby, J. 1969. Attachment and Loss. Trad. it. Attaccamento e perdita. Vol. 1: L'attaccamento alla madre. Torino: Bollati Boringhieri (1999).

Charmet, G. P. 2000. I nuovi adolescenti. Padri e madri di fronte a una sfida. Milano: Raffaello Cortina.

Di Summa, F. 2013. Essere protagonisti nella costruzione del benessere: famiglia e scuola in sinergia, in L. G. Grandi \& G.S. Lerda (eds.) Per $i$ nostri figli, pp. 75-84. Cuneo: Percorsi.

Erikson, E. 1950. Childhood and Society. Trad. it. Infanzia e Società. Roma: Armando (2008).

Gallese, V., Keysers, C., \& Rizzolatti, G. 2006. A Unifying View of the Basis of Social Cognition. Trends in Cognitive Sciences, 396-403.

Gallese, V. 2006. Intentional Attunement: A Neurophysiological Perspective on Social Cognition. Brain Res. Cog. Brain Res., 1079, 15-24.

Gallese, V. 2007. Dai neuroni specchio alla consonanza intenzionale: Meccanismi neurofisiologici dell'intersoggettività. Rivista di Psicoanalisi, 1, 197-208.

Grandi, L. G. \& Lerda, G. S. 2013. Per i nostri figli. Cuneo: Percorsi.

Grandi, L. G. 2002. Viaggio nell'uomo. Torino: Ideanet.

Grandi, L. G. 2016. Amore e psyche. Percorsi di psicoterapia individualpsicologica. Torino: Effatà Editrice.

Guidi, R., Fonović, K., \& Cappadozzi, T. 2017. Volontari e attività volontarie in Italia. Bologna: Il Mulino.

Lerda, G. S. \& Lo Sapio, V. 2014. The Precariousness of Relationships During the Age of the Digital Native. Aloma. Revista de Psicologia, Ciencies de l'Educaciò i de l'Esport, 32 (2), 77-83.

Lorenz, K. 1949. Er redete mit dem Vieh, den Vögeln und den Fischen. Trad. it. L'anello di Re Salomone. Milano: Adelphi (2003).

Mortari, L. 2006. La pratica dell'aver cura. Milano: Bruno Mondadori.

Recalcati, M. 2012. Ritratti del desiderio. Milano: Raffaello Cortina.

Rizzolatti, G. \& Sinigaglia, C. 2006. So quel che fai. Il cervello che agisce e $i$ neuroni specchio. Milano: Raffaello Cortina.

Siegel, D. J. 1999. The Developing Mind. Trad. it. La mente relazionale. Neurobiologia dell'esperienza interpersonale. Milano: Raffaello Cortina (2001).

Spitz, R. \& Cobliner, W. G. 1965. The first Year of Life: A Psychoanalytic Study of Normal and Deviant Development of Object Relation. Trad. it. Il primo anno di vita del bambino. Studio psicoanalitico sullo sviluppo delle relazioni oggettuali. Roma: Armando (1972).

Stern, D. N. 1985. The Interpersonal World of the Infant. Trad. it. Il mondo interpersonale del bambino. Torino: Bollati Boringhieri (1987).

Ternynck, C. 2011. L'homme de sable: Pourquoi l'individualisme nous rend malades. Trad. it. L'uomo di sabbia. Individualismo e perdita di sé. Milano: Vita e Pensiero (2012). 
Winnicott, D. 1951. Transitional Objects and Transitional Phenomena. Trad. it. Oggetti transizionali e fenomeni transizionali. Dalla pediatria alla psicoanalisi. Firenze: Psycho (1991). 


\title{
Gian Sandro Lerda
}

(Cuneo, Italy)

Legami capaci di futuro: dalle basi intersoggettive dello sviluppo della persona all'esperienza dello "stare" in relazione. Attaccamento, fiducia, desiderio e cura nei rapporti interpersonali

\begin{abstract}
English). Many conceptual analyses of the structure and mode of operation of the contemporary society outline a worrying and discouraging framework. They describe the decadence of values and they depict a world populated with individuals who are narcissistically selforiented, looking for the satisfaction of their own desires, unable to desire, build and keep stable, continual and deep relations and affects. Nevertheless, recent psychological research, supported by the evidence of neuroscientific research, has evidenced that the origins of the psychic development of the individual are connected to the domain of the relational experience. New-borns become persons in and thanks to the context of relations where they live and grown up. Thus, relations are an essential feature and a fundamental and peculiar experience for the human being. This is evident if we try to analyse aspects and dimensions, such as attachment, trust, desire and care.
\end{abstract} Attachment allows not only the survival but also the internalization of relational patterns which are necessary for life. The trust experience in the primal relations allows the vital energy to be addressed towards the world and to build ties thanks to the experience of faith in the other. Experimenting satisfaction and frustration, possibility and limits in the interpersonal relations fosters the development of desire and of the capability of wait, renounce, choice, care, as well as the necessity/opportunity of recognizing/knowing the other. Feeling himself or herself understood by a caring other prepares the ground for the experience of personal safety and thanks to some special pedagogical reinforcements, it opens to the possibility of taking care of someone else, in a structure of mutual exchanges and material, affective and spiritual support which is necessary for any human being. Significant socio-cultural changes occurred in the last decades have influenced those fundamental experiences and contributed to produce new relational styles and models which are characterised by precariousness and discontinuity. It is thus of primary importance to promote and sustain a reflection and education on these themes with the aim of fostering our needs and relations, whose expression is essential for the wellness and self-realization of individuals and communities. And this is not only in order to achieve a harmonic development of growing-up subjects but even for adults 
who want to fully accomplish their life and the related needs of sharing, intimacy and generativity.

These results have to be achieved by taking into consideration the peculiarity of modern life and by searching for creative solutions thanks to which, at least partially, we can try to combine the new claims with the essential experience of "being" in a relation.

Abstract (Italiano). Numerose analisi attuali circa l'assetto e il funzionamento della società contemporanea tratteggiano un quadro allarmante e scoraggiante, descrivono la decadenza di valori e riferimenti e rappresentano un mondo popolato da individui narcisisticamente ripiegati su di sé e orientati alla soddisfazione delle proprie voglie, incapaci di desiderare, costruire, mantenere legami e affetti dotati di stabilità, continuità e profondità. Eppure le discipline psicologiche, sostenute recentemente dalle scoperte nell'ambito delle neuroscienze, concordano nell'individuare nell'esperienza relazionale la base della nascita e dello sviluppo psichico dell'individuo: il neonato diventa persona all'interno e in virtù della rete di relazioni nella quale nasce, vive e cresce. La relazione è dunque presupposto imprescindibile, esperienza irrinunciabile, fondante e peculiare dell'uomo. Ciò risulta evidente provando ad analizzarne alcuni aspetti e dimensioni correlate: l'attaccamento, la fiducia, il desiderio e la cura.

L'attaccamento consente non solo la sopravvivenza, ma anche l'interiorizzazione di quei patterns relazionali indispensabili alla vita. L'esperienza della fiducia di base nelle relazioni primarie permette all'energia vitale di orientarsi verso il mondo e di costruire legami grazie alla possibilità di fidarsi e di affidarsi. Lo sperimentare soddisfazione e frustrazione, possibilità e limiti nei rapporti interpersonali sostiene lo svilupparsi del desiderio e delle capacità di attesa, rinuncia, scelta, impegno, così come la necessità/opportunità di riconoscere/conoscere l'altro. Il sentirsi compreso nello sguardo e nella mente di un altro che si prende cura pone le basi della sicurezza personale e apre, attraverso opportuni rinforzi educativi, alla possibilità di prendersi cura di altri, in quell'intreccio di reciproci scambi e sostegni materiali, affettivi e spirituali di cui l'uomo non può fare a meno.

I significativi mutamenti socio-culturali intercorsi negli ultimi decenni hanno senza dubbio influito su queste esperienze fondamentali, contribuendo a produrre nuovi stili e modelli relazionali, caratterizzati da precarietà e discontinuità. È quanto mai necessario promuovere e sostenere una riflessione e una formazione su questi temi per riprendere contatto e dare spazio ai nostri sani bisogni di relazione, la cui espressione è indispensabile per il benessere e la realizzazione individuale e comunitaria: non solo per uno sviluppo armonico dei soggetti in crescita, ma anche per l'individuo adulto che intenda accedere ad un percorso di vita rispondente alle sue istanze più profonde, connotate da bisogni di condivisione, intimità e generatività. 
Cio e da ricercarsi tenendo conto delle caratteristiche e peculiarita della vita moderna, attraverso soluzioni creative che consentano, almeno parzialmente, di coniugare le nuove esigenze con l'esperienza irrinunciabile di "stare" in relazione.

Keywords: ties, attachment, trust, desire, care

Parole chiave: legami, attaccamento, fiducia, desiderio, cura

Ethics in Progress (ISSN 2084-9257). Vol. 8 (2017). No. 1, Art. \#11, pp. 174-196.

Creative Commons BY-SA 3.0

Doi: 10.14746/eip.2017.1.11 